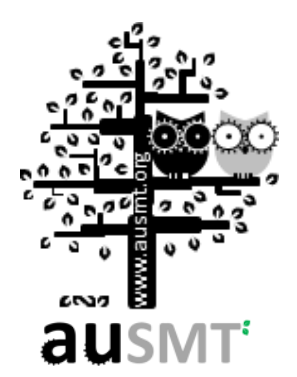

\title{
Responding to Industrial Transformation and Upgrade - New Concept for Intellectual Property Management Services Development in Taiwan
}

\section{Chih-Lun Chen*}

Market Intelligence \& Consulting Institute (MIC), Institute for Information Industry (III), Taiwan

(Received 27 November 2013; Published on line 1 December 2013)

*Corresponding author: llinahlli@micmail.iii.org.tw

DOI: 10.5875 /ausmt.v3i4.383

The International Institute for Management Development (IMD) has performed a five-year moving average calculation on major national productivity statistics for countries worldwide. The results indicated that the average rate of global productivity growth between 1999 and 2003 was 10.2\%, but the rate declined to $2.35 \%$ between 2008 and 2012. This trend shows that the momentum of development between nations has waned. Although companies have made large, multi-oriented, and highly-complex investments in innovation projects, innovations that inspire significant change worldwide and increase the speed of productivity are still difficult to produce. The current condition reflects the "Great Stagnation" perspective proposed by contemporary economics expert Tyler Cowen.

Continued poor economic conditions have affected Taiwan's industrial development despite the fact that evaluations from internationally renowned research institutions, such as the IMD and the WEF, have continued to affirm Taiwan's strengths in technological innovation and technological infrastructure. However, in-depth reviews still indicate that Taiwanese industries are currently experiencing issues such as technology and trade imbalance, decreases in manufacturer profit margin, and insufficient momentum. These obstacles must be dealt with in the future.

To face the "Great Stagnation" era, Taiwan has the advantages of knowledge, innovation, and intensive entrepreneurship. In addition to continuing its strong innovation capacities, broad interdisciplinary integration and new core industry technology creation are vital to turn this crisis into an opportunity. To help domestic enterprises adjust to the industry as a whole and expand the market, the government is actively promoting the "Three Industries, Four Reforms (incorporation of a service element by manufacturing firms, incorporation of advanced technology and international reach by service firms, and achievement of greater distinctiveness by firms in traditional sectors)" as well as the "Free Economic Pilot Zones (FEPZs)" to accomplish industry transformation and upgrade. The objective is to enhance the added-value of overall industries by upgrading industrial technology and substance as well as logistics and spatial resource arrangement in order to achieve service exportation, service industry modernization, and high-value business opportunities and markets.

Under the development of the manufacturing industry and integrated services industry, a review of the various policies in recent years indicated that companies are focusing upon patent and intellectual property management and services. From a practical standpoint, intellectual property management and patent implementation can solidify Taiwan's manufacturing foundation and quality innovation capacity. They are also the key to maintaining an advantage and to extending the value chain. Therefore, this study will focus on the developmental needs of Taiwan's intellectual property management services and propose the direction of strategic priorities for the future. 


\section{Taiwan Must Actively Enhance its Intellectual Property Management Capabilities}

The annual reports issued by the five largest patent offices in the world (IP5, which includes the United States, Japan, Europe, South Korea, and China) indicate that Taiwanese enterprises have been applying for patents very actively. For example, the number of patent applications filed by Taiwanese enterprises to IP5 ranked in the top 10 worldwide between 2011 and 2012. Most Taiwanese patent applications were filed to the patent offices in China and the United States, and the number of patents filed by Taiwan to these two patent offices ranked third and fourth compared to other nations worldwide. The results reveal that Taiwan's primary markets are China and the United States, and Taiwan has been laying the foundation for these two markets.

Enterprise patent application statistics from the United States Patent and Trademark Office (USPTO) in 2012 showed that Foxconn ranked eighth in the number of patent applications filed. However, it is noteworthy that among the top 10 enterprises, seven are from Taiwan, Japan, and Korea. This highlights the intensity of the patent competition between Taiwan and its neighboring countries.

Although Taiwan has strong innovation capacity and a large number of patent applications and approvals, the Central Bank's statistics indicate that there is still a multi-fold gap between Taiwan's patent and trademark royalty expenses and revenues. Using 2012 as an example, the total expenditure was up to US\$4.549 billion and the total revenue was only US\$932 million, a gap of US\$3.617 billion. Although this gap has diminished compared to the gap of $\$ 4.95$ billion in 2011 , there must be further planning to improve patent management, distribution, and implementation.

In addition, there are a growing number of international non-practicing entities (NPEs). They rely on the advantages of having a large amount of funding and patents to execute for-profit actions through operating models such as financial services, as well as technology integration, IP defense, or even lawsuits. General observation revealed that the number of patent litigation cases filed by NPEs in recent years has rapidly increased, but patent infringement suits filed by enterprises have

Mr. Chih-Lun Chen's research areas include industry technology screening and evaluation, blueprint planning of industrial technology development, and hometown development. Mr. Chen has participated in the projects of "Strategy Planning for the Core Industries' Development of New Taipei City", and "Cross-Industry Cooperation in Free Economic Pilot Zones (FEPZs)", responsible for industry development strategies of New Taipei City, and the research of intellectual property industry. Mr. Chen holds a Master's degree in geography from National Taiwan University. declined each year. Regarding Taiwanese corporations, NPEs have filed 106 patent lawsuits against HTC in the last 5 years, ranking seventh worldwide in the number of lawsuits filed against a company, while ASUS and Acer rank 22nd and 26th, respectively. This indicates that Taiwanese enterprises have an urgent need for patent layout and defense.

Taiwan is afflicted with the various issues summarized above. In order for Taiwanese patents and intellectual property to realize their full potential, we believe that Taiwan should focus on "patent portfolio and layout recommendations", "assistance for patent defense and counterclaims", and "commission foreign and domestic specialists to execute patent drafts and applications" as priorities to actively improve the intellectual property management services layout.

\section{Taiwan Must Develop Intellectual Property Management Services with Innovative Thinking}

The Board of Science and Technology, Executive Yuan passed the Taiwan Intellectual Property Strategic Program in October 2012. The program's vision is to establish Taiwan as an Asia-Pacific intellectual property creation and application powerhouse. Main objectives include expanding the intellectual property adoption value, enhancing the strength of intellectual property protection, and providing an extensive intellectual property infrastructure. Furthermore, the government has established an intellectual property bank (IP Bank) through corporate capacity to achieve the primary goals of establishing intellectual property joint defense mechanisms for domestic enterprises as well as providing professional advisory and strategic cooperation services for patent agency representation, patent invalidation, joint negotiation, and intellectual property litigation. Unfortunately, domestic industries are still accustomed managing their own intellectual property due to a lack of operational funds, which is hindering the development of the IP Bank.

Taiwan must overcome significant difficulties if it wants to attract international intellectual property management companies to establish stations and provide services in Taiwan. There is no direct incentive for large intellectual property management service companies to set up stations in Taiwan due to the small size of Taiwan's market and the country's territorialist intellectual property application model. In terms of layout access for other markets, Taiwan must cultivate intellectual property research, draft, and language talent as well as improve its understanding of foreign patent applications, backgrounds, and cultures. In addition, 
most international intellectual property management companies already have a comprehensive and dense global network layout. Some markets have elected to cooperate with local patent brokers, so there is no need to directly establish stations in Taiwan. Therefore, if Taiwan wants to develop intellectual property management services at this stage, it must adopt strategic thinking that is different from the past.

\section{Singapore Promotes the Vision of an Asian Global Intellectual Property Center through Specialization and Internationalization}

The mistakes of others can reveal solutions. Singapore and Taiwan are on the periphery of the major Asia market and both have relatively small populations. Singapore has the advantages of being multi-lingual, multi-ethnic, and maintains a neutral and friendly position among major economic powers such as China, India, and Japan, in addition to being located at the geographical center of Southeast Asia. Furthermore, Singapore's government provides a comprehensive mechanism for IP protection and providing assistance for patent application in other countries. In 2013, Singapore proposed a vision of becoming the global intellectual property center for Asia. The vision's primary objectives and measures include:

1. Becoming a center for intellectual property dispute resolution: Adopt a positive grant system for patents that is consistent with those of Europe and the United States. All of the applications must pass the actual research and review procedure, and cases with unresolved patentability will be rejected. Meanwhile, Singapore is collaborating with the World Intellectual Property Organization's Arbitration and Mediation Center (WIPO AMC) so that litigants may select a trustworthy third-party with relevant expertise and experience from the WIPO AMC group to participate in the arbitration processes, which can save litigation time and costs.

2. Becoming a center for intellectual property applications: Reduce the time and costs required for the patent registration by collaborating with the official IP Academy and WIPO, invest SG\$50 million to cultivate patent research, review, and drafting of talent, and establish Patent Prosecution Highways (PPH) with the United States and Japan. In addition, the government and patent offices have thoroughly prepared the data, windows, and models needed to assist international and domestic enterprises in applying for foreign country patents as well as to facilitate enterprises to apply for foreign IP applications.

3. Becoming a center of intellectual trade and management: Encourage enterprises to establish regional headquarters and bring patents into Singapore with incentives such as tax reliefs, low-income tax policies, and provide assistance for IP Protection as well as foreign and domestic IP applications. Furthermore, the Intellectual Property Office of Singapore (IPOS) has established a Centre of Excellence for IP Valuation to conduct R\&D for IP evaluation related methods in cooperation with European and American professional intellectual property evaluation companies in order to develop IP financing businesses with domestic financial and manufacturing sectors.

Singapore's experience indicates that professional training and an international infrastructure are vital for comprehensive IP management service operations anticipating to expand into the international market. Taiwan still has the opportunity to develop its intellectual property services industry if it can incorporate these intellectual property management development concepts.

\section{Use New Talent Cultivation, Market Expansion, and Creative Industry Concepts to Develop Intellectual Property Management and Drive Domestic Industry Transformation and Upgrade}

As discussed in the previous section, Taiwan must provide overall strategic planning in terms of personnel training, market expansion, and creative industries to satisfy key intellectual property service demands that include "patent portfolio and layout recommendations", "patent defense and counterclaim assistance", and "commission foreign and domestic specialists to execute patent drafts and applications". In the following section, we will adopt the "turning stone into jade" approach to propose conceptual ideas that can serve as a reference for the relevant reform departments.

First, in terms of talent cultivation, Taiwan has a very limited number of professional patent researchers and drafters who are familiar with foreign market conditions and can provide assistance with foreign patent applications. However, the WIPO already provides patent personnel training services. If a country or department were to file an application, the WIPO will 
select a retired personnel of the organization to provide training assistance at the application country. This method can rapidly enhance talent specialization and internationalization.

From the perspective of assisting domestic industries to perform patent layout, numerous domestic patent drafts or acquisitions have reduced patents content and function or provided ineffective defense capabilities in order to save time, decrease costs, or reduce the number of questioning and answering sessions required. This approach is a further waste of resources. Others have duplicated patent portfolios needlessly with insufficient core patent components. Not only is this situation a waste of application costs, it also cannot prevent litigations. In sum, establishing a sound and robust domestic intellectual property management system or even a professional service industry can help the domestic industry to reduce the associated costs.

In terms of overseas market development, we recommend that the Intellectual Property Office, national patent offices, and other related industries gradually establish roots in overseas markets and establish PPHs with various countries. It is especially critical for talent, models, and programs to be able to readily provide assistance for patent applications in other Asian countries so that enterprises may be more willing to headquarter in Taiwan and expand their mark overseas.

In addition, in terms of patent offices and domestic industries related intellectual property, their accumulated litigation experience, international network connections, and high-quality and affordability compared to foreign firms will undoubtedly have a considerable appeal to middle and small sized enterprises with limited funds that are seeking assistance for foreign litigation and patent drafting. The development of such business outsourcing services not only can maintain and strengthen the core competencies of Taiwan's own intellectual property management related services, they can also help expand their client base to foreign enterprises and achieve a service exportation effect.

As a medium- to long-term goal, Taiwan has the ability to develop the next generation of new products or even new industries by incorporating its innovative and entrepreneurial capacities, high-quality manufacturing, patent commercialization, and production capacity with assistance from professional organizations or service companies in patent portfolio and product development. To meet patent as well as domestic and international $R \& D$ demands, foreign talent must be introduced, and an innovative customs mechanism must be provided. Also, the three high-quality environments-ecology, life, and products-must be afforded to create and gradually upgrade the various hardware and software facilities needed to promote a comprehensive industrial transformation and further provide an enhanced operating environment under the spirit of "Three Industries, Four Reforms". 